\title{
Managing the risk and benefits of digital technologies in social assistance provision
}

\author{
Dr Becky Faith and Dr Tony Roberts
}

February 2022

\section{Theme summary}

Aid agencies, governments, and donors are expanding investment in the digitisation of their beneficiary identification and registration systems, in digitised systems for cash payments, and in the remote and algorithmic control of humanitarian and social protection programmes. This is happening in ways that may facilitate the move from humanitarian assistance to government provision and may enable the delivery of shockresponsive social protection. Yet humanitarian and social protection actors are increasingly concerned about a range of risks and accountability vacuums associated with the adoption of these

\begin{abstract}
technologies. While claims for the benefits of digitisation often rest on cost savings, data relating to these costs and benefits are not easily accessible. There is also an urgent need to adopt approaches to value for money in this sector that recognise the digital dignity of beneficiaries. A knowledge gap exists around how the movement towards biometric identification and algorithmic management using humanitarian and social protection data will affect the interests of vulnerable populations - so too does a gap in research that is focused on the standpoints, interests, and priorities of these populations.
\end{abstract}

This thematic brief is a shortened version of a BASIC Research Working Paper. To explore this research theme in more detail please refer to:

Faith, B.; Roberts, T. and Hernandez, K. (2022) Risks/Accountability/Technology Thematic Working Paper, BASIC Research Working Paper 3, Brighton: Institute of Development Studies, DOI: 10.19088/BASIC.2022.003

A full list of the references cited in this brief can be found at the BASIC Research Zotero library. Implemented by
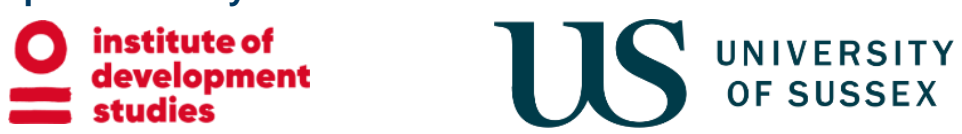

UNIVERSITY OF WOLVERHAMPTON

\section{CIDT}

Better Assistance in Crises (BASIC) Research (funded by UKAid) aims to inform policy and programming on how to help poor and vulnerable people cope better with crises and meet their basic needs through more effective social assistance. All costs related to BASIC Research are covered by the UK Foreign, Commonwealth and Development Office. 


\section{State of the evidence and debate}

- Digitised systems have the potential to reduce transaction costs, enable real-time analysis and deliver affordances of scale and efficiency for humanitarian actors and governments. These include the potential to serve communities in hard-to-reach areas through electronic cash transfers and 'remote' programming which reduce security risks for donors and recipients.

- These systems also bring risks of exclusion for digitally disconnected populations and of rights violations for already vulnerable people. Such risks are more likely given the rapid adoption of untested technologies by unaccountable actors, in an absence of regulation.

- Humanitarian actors have launched a range of initiatives aimed at addressing these harms through the ethical use of data-driven technologies. There has been significant activity in this space including toolkits and handbooks aimed at integrating data protection principles and rights in the humanitarian environment. New resources have been released by the Cash Learning Partnership (CaLP) to support cash and voucher assistance (CVA) practitioners in working data responsibility into all aspects of their programmes. In the social protection sector, programmes have expanded with little consideration of these issues.

- The use of digital technologies and the pressure to innovate can be at odds with the precautionary principle; it is of vital importance that the voices and interests of affected populations remain central.

\section{Gaps in the evidence}

- Research is needed to better understand how political and commercial drivers, the pressures of crisis contexts, and the desire to move to government provision are all shaping the adoption of digital technologies. Research is also required to understand the opportunities, risks, and implications for accountability upwards to donors but also downwards to affected populations.

- There is an overall research gap and a lack of comparative studies that explore whether the processes of digitalisation and datafication of social assistance systems enable or inhibit accountability and inclusion. Specifically, there are key issues relating to informed consent and data sharing, the digital threat landscape in conflict settings, and the true value of predictive analytics in improving issues such as targeting in the humanitarian/social protection field.

- We also need to understand in greater depth how digital technologies can address power asymmetries by making humanitarian and government systems more accountable and transparent to affected populations, or by powering decentralised systems to allow people to retain agency over their own digital identity and service user information. 


\section{Directions for research}

- Addressing those gaps suggests an agenda for BASIC research on these issues that is focused on the standpoints, interests and priorities of affected populations. This would build their understanding and the understanding of humanitarian and social protection staff about the implications of this rapidly evolving technological environment.

- There are important issues to be investigated relating to the transparency of the claimed financial benefits of digitised systems; this would enable a fresh conceptualisation of what value for money should mean in this sector. To achieve this there is a need for research mapping of corporate actors in digital registration, identity, artificial intelligence, and remote monitoring. The dimensions of the market, the scale or direction of the investments being made, or the drivers, key actors, or tools are all poorly understood. Data on these would be important in assessing the process of privatisation and the implications for the move from humanitarian provision to government provision.

- While both social protection and humanitarian actors have many laudable initiatives regarding data responsibility in the CVA field, there is a lack of research about how this guidance is being applied in practice. Similarly, although guidance exists on the digital enablers and the ecosystem approach required to transition to national social protection systems from humanitarian assistance, this transition is not a linear one-way progression. Consequently, further research is required to understand the political and privacy challenges of integrating datafied systems, particularly in politically sensitive contexts.

\section{Acknowledgements and Disclaimer}

This document was developed by the Better Assistance in Crises (BASIC) Research programme. BASIC is implemented by the Institute of Development Studies (IDS), the University of Sussex and the Centre for International Development and Training, funded by UKAid from the UK government. The views expressed in this document are entirely those of the authors and do not necessarily represent views or policies of the UK governments official policies.

(C) IDS copyright 2022. Copyright in the typographical arrangement and design rests with IDS.

This publication (excluding the logos) may be reproduced free of charge in any format or medium, provided that it is reproduced accurately and not used in a misleading context. The material must be acknowledged as IDS copyright with the title and source of the publication specified.

Published by IDS.

DOI: 10.19088/BASIC.2022.025 